\title{
Influence of sulphasalazine, methotrexate, and the combination of both on plasma homocysteine concentrations in patients with rheumatoid arthritis
}

Cees J Haagsma, Henk J Blom, Piet L C M van Riel, Martin A van't Hof, Belinda A J Giesendorf, Dinny van Oppenraaij-Emmerzaal, Levinus B A van de Putte

Department of Rheumatology C J Haagsma P L C M van Riel L B A van de Putte

Laboratory of Paediatrics and Neurology H J Blom B A J Giesendorf $\mathrm{D}$ van

Oppenraaij-Emmerzaal

University Hospital Nijmegen, the Netherlands

Department of Statistical

Consultation, University of Nijmegen, the Netherlands M A van't Hof

Correspondence to: Dr C J Haagsma, Department of Rheumatology, University Hospital Nijmegen, PO Box 9101, 6500 HB Nijmegen, the Netherlands.

Accepted for publication 13 October 1998

\begin{abstract}
Objective-To study the influence of sulphasalazine (SSZ), methotrexate (MTX), and the combination (COMBI) of both on plasma homocysteine and to study the relation between plasma homocysteine and their clinical effects.

Methods-105 patients with early rheumatoid arthritis (RA) were randomised between SSZ (2-3 g/day), MTX (7.5-15 mg/week), and the COMBI (same dose range) and evaluated double blindly during 52 weeks. Plasma homocysteine, serum folate concentrations, and vitamin B12 were measured. The influence of the C677T mutation of the enzyme methylenetetrahydrofolatereductase (MTHFR) gene was analysed.

Results-A slight trend towards increased efficacy and an increased occurrence of minor gastrointestinal toxicity was present in the COMBI group, no differences existed clinically between SSZ and MTX. Only a slight and temporary increase in plasma homocysteine was found in the SSZ group, in contrast with the persistent rise in the MTX group and the even greater increase in the COMBI patients. Patients homozygous for the mutation in the MTHFR gene had significantly higher baseline homocysteine, heterozygous MTHFR genotype induced a significantly higher plasma homocysteine at week 52 compared with no mutation. No correlation was found between clinical efficacy variables and homocysteine. Patients with gastrointestinal toxicity had a significantly greater increase in homocysteine.

Conclusion-A persistent increase in plasma homocysteine concentrations was observed in patients treated with MTX alone and more pronounced in combination with SSZ, in contrast with SSZ alone. An increase in plasma homocysteine is related to the C677T mutation in MTHFR. A relation in the change in homocysteine concentrations with (gastrointestinal) toxicity was found, no relation with clinical efficacy existed.

(Ann Rheum Dis 1999;58:79-84)
\end{abstract}

The pharmacotherapy of rheumatoid arthritis (RA) consists of first line agents (non-steroidal anti-inflammatory drugs) and second line agents, also referred to as disease modifying anti-rheumatic drugs. The latter drugs generally have a slow onset of action. Favourable exceptions with a relatively rapidly occurring clinical effect are methotrexate (MTX) and sulphasalazine (SSZ). ${ }^{1}$ MTX is a well known anti-metabolite with dihydrofolate reductase inhibition as the primary target (see fig 1 ). ${ }^{2}$ SSZ has also been mentioned to have antifolate properties, demonstrated only in vitro on dihydrofolate reductase. ${ }^{3}$ The pharmacologically active ${ }^{4}$ metabolite of SSZ, sulphapyridine, however, does not have such properties. ${ }^{35}$ In some in vivo studies, SSZ was found to influence folate concentrations, possibly by interference with folate absorption. ${ }^{5}$ The influence of SSZ in RA on serum folic acid concentrations has been disputed. ${ }^{6}$ Direct influence on the folate (dependent) metabolism in vivo has not been investigated yet and the influence of folates on the clinical impact of SSZ has never been studied. In RA there is a clear folate related influence of MTX on toxicity and possibly on efficacy. Folinic acid in relatively high doses to circumvent the blockade of dihydrofolate reductase by MTX, resulted in an increased activity of the arthritis, suggesting that efficacy could at least partly be mediated by folate dependent ways. ${ }^{78}$ Folic acid, when given in low or higher doses $^{9}$ and also low dose folinic $\operatorname{acid}^{10-13}$ have favourably influenced MTX toxicity. Although sample sizes were possibly too small to definitely prove the absence of influence on efficacy, most and the largest of the above mentioned studies reveal no influence on efficacy of MTX. Timing and dose of folinic acid probably also play a part in this matter.

The inhibition by MTX of folate metabolism can interfere with purine and thymidine synthesis but also with transmethylation reactions by S-adenosylmethionine, influencing substances involved in inflammation, for example, adenosine and polyamines. ${ }^{14} 15$

The combination of SSZ and MTX was found to be more effective in RA patients with insufficient efficacy of SSZ, than MTX alone. ${ }^{16}$ The question is raised whether this has to do with the influence of both drugs on folate related metabolism, analogous to the synergism of sulphamethoxazole and trimethoprim in treating bacterial infections.

A way to measure the influence on folate related cellular metabolism in vivo is to measure plasma homocysteine. ${ }^{17}$ Possibly these 


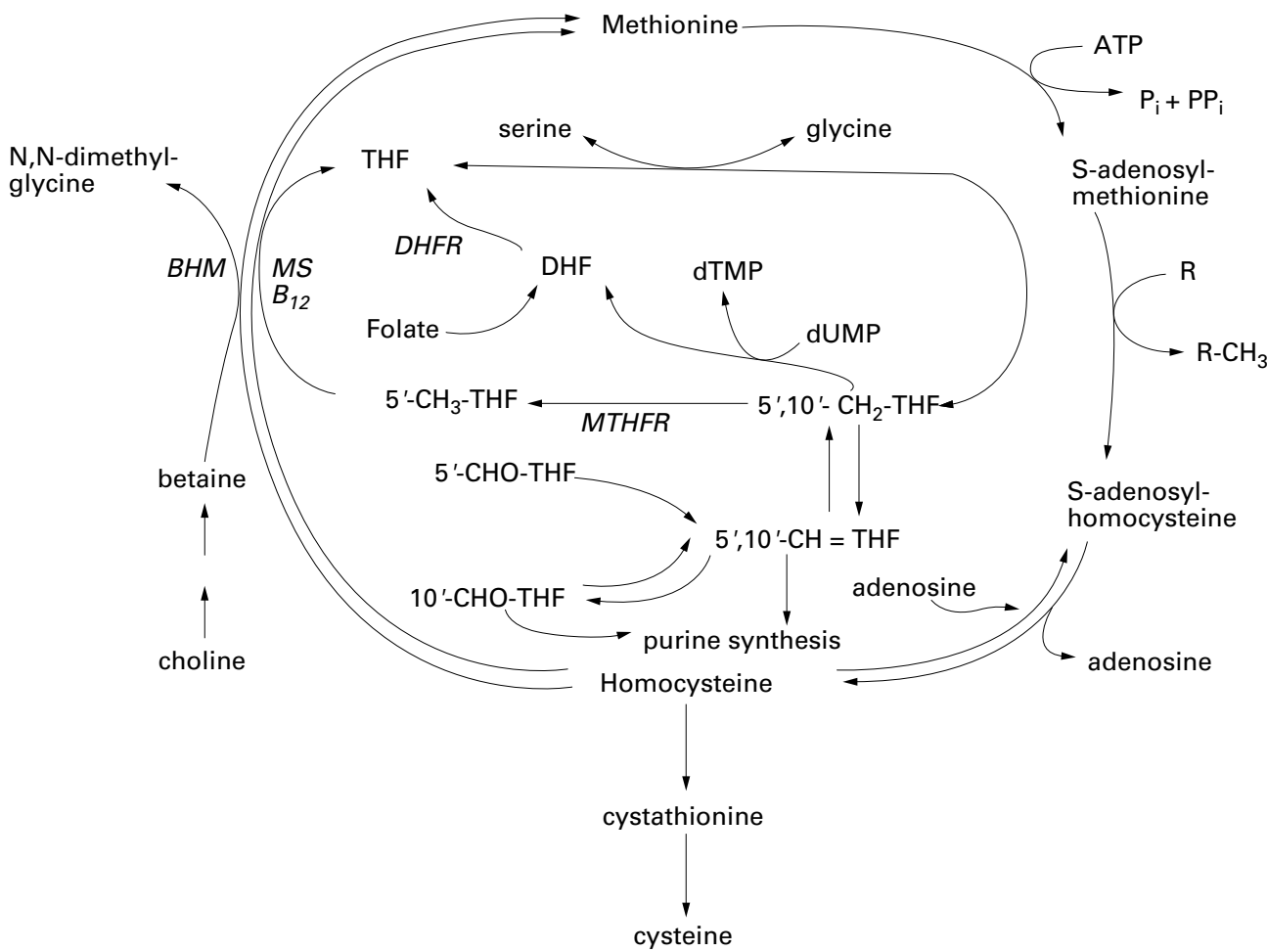

Figure 1 Folate metabolism. DHF: dihydrofolate, THF: tetrahydrofolate, 5',10-'CH$-\mathrm{CHF}$ : methylene-THF,

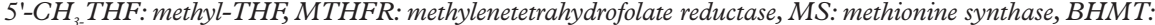
betaine-homocysteine methyl transferase, B12: vitamin B12.

changes in plasma homocysteine could be more pronounced, when the folate dependent metabolism is stressed in people with a mutation of genes of enzymes involved in folate metabolism. A C677T polymorphism of the enzyme methylene-tetrahydrofolate reductase (MTHFR), the homozygous mutation occurring in about $10 \%$ of the general Dutch population, ${ }^{18}$ has been shown to have influence on the reduction of 5,10-methylenetetrahydrofolate to 5-methyltetrahydrofolate, which is the methylgroup donor of the re-methylation of homocysteine to methionine. ${ }^{19}$ This metabolic stress could be produced by dihydrofolate reductase antagonists like MTX, or SSZ. The latter has been shown to inhibit MTHFR in vitro. ${ }^{3}$ Furthermore, the C677T mutation leads to redistribution of folate derivatives ${ }^{20}$ possibly leading to altered clinical effects of folate influencing medication.

In this randomised clinical trial comparing SSZ, MTX, and the combination, we studied whether these three medications influence plasma homocysteine concentrations, being an indication of interference with folate dependent metabolism. In this respect the role of the C677T mutation in the MTHFR gene, and the relation between plasma homocysteine and clinical efficacy and toxicity of these drugs was also studied.

\section{Methods}

One hundred and five patients with active early RA satisfying the ACR classification criteria, rheumatoid factor positive and/or HLA DR1/4 positive, were included in the study. Patients with a folate or vitamin B12 deficiency were excluded. They participated in an investigation examining the efficacy and toxicity of SSZ, MTX, and the combination of both (COMBI). The design was a double blind, double dummy, controlled, randomised clinical trial in a setting of one academic and five non-academic rheumatology departments. Patients were randomised between SSZ 2000 mg daily, or MTX $7.5 \mathrm{mg}$ weekly, or the COMBI. In case of insufficient efficacy, the dose could be raised from week 16 onwards, in a single step (SSZ $3000 \mathrm{mg} /$ day, MTX $15 \mathrm{mg} /$ week or matching placebo). In case of folic acid deficiency and adverse events addition of folic acid was permitted. All patients were evaluated every two weeks for the first four weeks and every four weeks thereafter by one observer for 52 weeks. Primary evaluation criterion was the mean change in the Disease Activity Score (DAS) over time. The DAS consists of the Ritchie articular index, which is a graded score of the number of painful joints, the number of swollen joints, and the erythrocyte sedimentation rate $(\mathrm{ESR}) .^{21}$ Other criteria were the general well being expressed by the patient on a visual analogue scale, the occurrence of clinically apparent adverse events, and abnormal laboratory measurements: cytopenias, deterioration in glomerular filtration rate (serum creatinine $>1.5$-fold baseline creatinine), and liver enzyme increases (alkaline phosphatase, transaminases, and $\gamma$-glutamyltransferase). Adverse events in general and the adverse events possibly/probably attributable to the study medication were analysed by overall occurrence and occurrence by body system.

Blood was obtained from fasting patients at a standardised time in the morning, 24 hours after intake of the study medication. Plasma 
Table 1 Change in efficacy variables, means (95\% CI)

\begin{tabular}{llll}
\hline \multirow{2}{*}{ Variable } & \multicolumn{2}{l}{ Change from baseline to week 52} & COMBI \\
\cline { 2 - 4 } & SSZ & MTX & $-2.3(-2.7,-1.9)$ \\
\hline DAS & $-1.8(-2.3,-1.3)$ & $-2.0(-2.4,-1.7)$ & $-14.3(-17.3,-11.4)$ \\
Number of swollen joints & $-9.2(-12.2,-6.3)$ & $-12.4(-15.4,-9.5)$ & $-10.6(-12.5,-8.7)$ \\
Ritchie articular index & $-9.2(-11.7,-6.8)$ & $-9.5(-11.6,-7.5)$ & $-16.9(-20.4,-13.5)$ \\
Number of painful joints & $-12.5(-15.9,-9.1)$ & $-15.2(-18.2,-12.2)$ & $-20.6(-27.6,-13.7)$ \\
VAS general health (mm) & $-15.4(-25.8,-5.0)$ & $-21.3(-30.2,-12.3)$ & $-28(-37,-19)$ \\
ESR (mm/h) & $-17(-26,-8)$ & $-21(-28,-15)$ & .
\end{tabular}

MTX: methotrexate, COMBI: combination of methotrexate and sulphasalazine, SSZ: sulphasalazine, DAS: Disease Activity Score, VAS: Visual Analogue Scale $(0-100 \mathrm{~mm})$.

homocysteine was measured by HPLC, ${ }^{22}$ serum folate and vitamin B12 were measured by radioassay ${ }^{23}$ both at week 0 , and folate also at weeks $4,8,24$, and 52 . DNA extracted from the patient's white blood cells was screened for the C677T mutation of the MTHFR gene by polymerase chain reaction (PCR) and restriction enzyme analysis. ${ }^{14}$

\section{STATISTICAL ANALYSIS}

Continuous variables were evaluated by analysis of variance, multiple regression, and $t$ tests. The Tukey test was used in case of multiple comparisons. When variables were not normally distributed, a logarithmic or square root transformation was performed. The values of patients who ended the study prematurely were taken in account until drop out.

The correlation of changes in plasma homocysteine concentration and the value of the clinical variables (see above) calculated as an area under the time curve (week 0-52) was expressed by Pearson correlation coefficients. Changes between plasma homocysteine and serum folate at baseline and during the adverse event of patients having an adverse event were compared with changes of patients without an adverse event in the same period, analysed by one way analysis of variance. If a patient had several periods without adverse events, one of these periods was randomly selected. Thus, patients were thus used only once in the analysis. The total number of adverse events as well as the number of adverse events per body system were analysed for the different time intervals. The differences in baseline plasma homocysteine, serum folate, and vitamin B12 of patients with and without an adverse event were analysed by $t$ tests on log transformed values.

\section{Results}

One hundred and five patients participated in this study, 34 in the SSZ group, 35 in the MTX group, and 36 in the COMBI group. There

Table 2 Adverse events (AE), number of patients*

\begin{tabular}{lccl}
\hline & $S S Z$ & MTX & COMBI \\
\hline Total number of patients & 34 & 35 & 36 \\
Any AE & 30 & 27 & 32 \\
Possible/probable & 16 & 11 & $23 \ddagger$ \\
Nausea & 10 & 9 & $23 \dagger$ \\
Abdominal pain/discomfort & 9 & 7 & 13 \\
Increase in transaminases $>2 \times$ normal & 4 & 5 & 2 \\
Haematological & 1 & 1 & 0 \\
Neuropathy & 1 & 0 & 1 \\
Rash & 5 & 2 & 0
\end{tabular}

$\star$ One patient can contribute more than once. $t \mathrm{p}=0.002\left(\chi^{2}\right.$ test). $\neq \mathrm{p}=0.023\left(\chi^{2}\right.$ test $)$ were no significant differences in baseline characteristics between the three treatment groups. The mean age was 56 (SD 13) years, the number of female patients 68 , the number of male patients 37 . The mean disease duration was 2.9 (SD 1.9) months. Five patients were rheumatoid factor negative, 100 patients had rheumatoid factor positivity.

The baseline concentrations of plasma homocysteine, folate, and vitamin B12 were not different in the three treatment groups (see table 3). There was a significant correlation between baseline serum folate and baseline plasma homocysteine (Pearson's $r=0.47$, $\mathrm{p}<0.001)$. Z scores of the plasma homocysteine concentrations before drop out demonstrated that this event occurred randomly with respect to plasma homocysteine concentrations.

The clinical results are given in tables 1 and $2 .^{24}$ There were no statistically significant differences in efficacy between the treatment groups (one way analysis of variance). The combination group tended to have a better result. However, in that group significantly more patients experienced nausea (table 2). Eleven patients in the SSZ group, 11 in the MTX group, and 7 in the COMBI group had an increase in dose of the study medication (not significant). Folic acid supplementation was given to two patients in the SSZ group, to one in the MTX group, and to three in the COMBI group, measurements were taken in account until the start of folic acid.

The two treatment groups receiving MTX showed a persistent increase in homocysteine, in contrast with the SSZ group, which did not show a persistent change; only in weeks 4 and 8 there was a statistically significant but slight increase in plasma homocysteine (table 3 and fig 2). This increase of plasma homocysteine by MTX with or without SSZ was statistically significant both compared with baseline value and with SSZ. There was no statistically significant influence of the dose on plasma homocysteine. MTX with or without SSZ had a statistically significant influence on the change in serum folate concentrations for all weeks (4-52). SSZ had no influence on folate concnetrations. Multiple regression analysis showed that serum folate explained some of the variation in plasma homocysteine at week 24 and 52 only $(\beta-0.38$ and $-0.54, p<0.05)$, but also that MTX with or without SSZ had a statistically significant independent influence at all weeks ( $\beta$ between 0.21 and 0.28 , p values $<0.05)$. The rise of plasma homocysteine because of the combination of MTX and SSZ was greater than the rise 


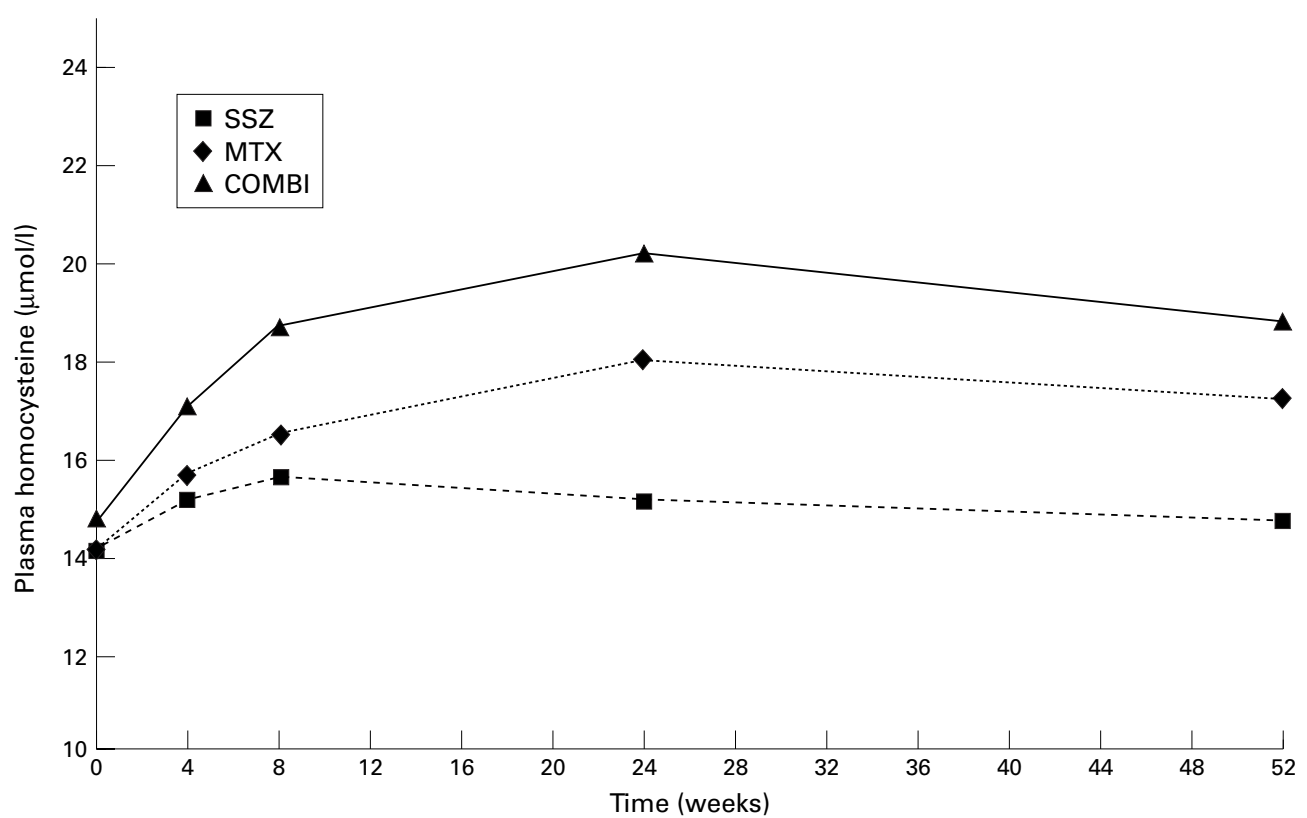

Figure 2 Plasma homocysteine compared with time. SSZ: sulphasalazine, MTX: methotrexate, COMBI : SSZ + MTX.

because of MTX alone although this difference did not reach statistical significance $(\mathrm{p}>0.1)$. The distribution of the different genotypes of MTHFR (no mutation (CC-genotype/ wildtype), heterozygous (CT), and homozygous (TT)) was as follows: SSZ: 12, 18, and 4; MTX: 17, 17, and 1; COMBI: 12, 18, and 5 respectively $(p>0.2)$. In table 4 the relation between the polymorphism of the MTHFR gene (CC, CT, TT) and plasma homocysteine is given. The percentage of patients in this study with a TT mutation of the MTHFR gene is not different from the general Dutch population. ${ }^{14}$ Homozygous patients had significantly higher baseline homocysteine concentrations compared with heterozygous patients and patients without the mutation. There was an influence of the genotype on plasma homocysteine discernable: at week 52 , patients with the CT mutation had significantly higher plasma homocysteine concentrations than patients with no mutation $(\mathrm{p}<0.05)$. The change from baseline to week 52 in the heterozygous patients (see table 4) was more pronounced than in the group of patients without the mutation, but the difference was not significant $(p=0.06)$. The difference between the homozygous patients and the other groups was no longer significant at week 52 . There was no significant interaction between the study medication and the MTHFR genotype concerning homocysteine concentrations at week 52 in the analysis of variance. This makes different effects of SSZ, MTX or COMBI on plasma homocysteine in the presence of different states of this enzyme less likely.

No consistent correlation between clinical efficacy variables (Ritchie articular index, number of swollen joints, general health as scored by the patient, and ESR) and plasma homocysteine was found (Pearson correlation coefficients $<0.3$, $\mathrm{p}$ values $>0.05$ )

Adverse events were analysed both as any adverse event and possibly/probably attributable to the medication. A rise in plasma homocysteine (an increase of $17 \%$, SEM $4.4 \%$ ) was found in patients experiencing a gastrointestinal adverse event (nausea, abdominal discomfort/pain, and heartburn taken together) without having other concommittant (a possibly confounding) adverse events. This rise was statistically significantly higher than the rise in plasma homocysteine of patients without an adverse event $(p<0.05)$. There was no statistically significant difference between patients having other adverse events compared

Table 3 Plasma homocysteine ( $\mu$ molll), serum folate (nmol/l), and vitamin B12 (pmol/l) and differences with week 0

\begin{tabular}{|c|c|c|c|c|c|c|c|c|c|c|c|}
\hline & \multicolumn{3}{|l|}{$W k 0$} & \multirow{2}{*}{$\begin{array}{l}\text { Wk } 4 \\
\text { Hcys }\end{array}$} & \multirow{2}{*}{$\frac{-W k 0}{\text { Folate }}$} & \multirow{2}{*}{$\begin{array}{l}W k 8 \\
\text { Hcys }\end{array}$} & \multirow{2}{*}{$\frac{-W k 0}{\text { Folate }}$} & \multirow{2}{*}{$\begin{array}{l}\text { Wk } 24 \\
\text { Hcys }\end{array}$} & \multirow{2}{*}{$\frac{-W k 0}{\text { Folate }}$} & \multirow{2}{*}{$\begin{array}{l}\text { Wk } 52 \\
\text { Hcys }\end{array}$} & \multirow{2}{*}{$\begin{array}{l}-W k C \\
\text { Folate }\end{array}$} \\
\hline & Hcys & Folate & vit $B 12$ & & & & & & & & \\
\hline SSZ, n & 34 & 34 & 34 & 31 & 31 & 30 & 30 & 26 & 26 & 22 & 22 \\
\hline Median & 14.1 & 10.0 & 205 & 1.1 & 0.0 & 1.5 & -0.5 & 1.0 & -0.1 & 0.6 & 0.2 \\
\hline P25 & 12.1 & 6.7 & 158 & 0.6 & -1.8 & 0.2 & -2.0 & -0.5 & -1.6 & -1.1 & -1.9 \\
\hline P75 & 17.1 & 12.0 & 287 & 2.5 & 1.0 & 2.7 & 1.3 & 3.0 & 1.7 & 2.7 & 2.9 \\
\hline MTX, n & 35 & 35 & 35 & 35 & 35 & 35 & 35 & 33 & 33 & 33 & 33 \\
\hline Median & 14.1 & 9.5 & 220 & 1.6 & -2.2 & 2.4 & -2.4 & 3.9 & -2.9 & 3.1 & -3.0 \\
\hline P25 & 12.1 & 8.4 & 170 & 0.9 & -3.0 & 0.5 & -3.5 & 1.1 & -4.4 & 0.5 & -4.3 \\
\hline P75 & 16.2 & 13.0 & 260 & 3.1 & -1.1 & 4.1 & -0.4 & 5.1 & -1.3 & 5.8 & -1.7 \\
\hline COMBI, n & 36 & 36 & 36 & 35 & 35 & 34 & 34 & 32 & 32 & 30 & 30 \\
\hline Median & 14.8 & 8.7 & 210 & 2.3 & -1.5 & 3.9 & -2.3 & 5.4 & -2.5 & 4.0 & -2.0 \\
\hline P25 & 12.5 & 6.5 & 170 & 1.2 & -3.0 & 0.8 & -3.0 & 3.7 & -4.3 & 2.0 & -4.7 \\
\hline P75 & 16.8 & 11.0 & 340 & 4.8 & -0.2 & 5.7 & -0.8 & 8.7 & -0.7 & 7.6 & -0.7 \\
\hline
\end{tabular}

SSZ: sulphasalazine, MTX: methotrexate, COMBI: SSZ+MTX, Hcys: plasma homocysteine concentration. Normal value of of serum folate: $>5.0$ nmol/1, vitamin $\mathrm{B} 12:>150 \mathrm{pmol} / \mathrm{l}$. 
Table 4 Relation between methylenetetrahydrofolate reductase (MTHFR) genotype and plasma homocysteine, mean/median (P25, P75)

\begin{tabular}{llllll}
\hline MTHFR & $n$ & Hcyst week 0 & $n$ & Hcyst week 52 & p value $\neq$ \\
\hline 0 & 40 & $13.6 / 13.9(11.8,15.7)$ & 31 & $15.1 / 14.2(12.9,19.1)$ & $<0.05$ \\
1 & 53 & $14.6 / 14.6(12.2,17.0)$ & 44 & $18.4 / 18.3(14.5,23.0)$ & $<0.05$ \\
2 & 10 & $19.6 / 17.7(14.4,24.5)$ & 9 & $19.4 / 21.0(17.7,23.3)$ & $>0.05$ \\
p value & & $<0.05,2$ v 1 and 0 & & $<0.05,2$ and 1 v 0 & \\
\hline
\end{tabular}

$\star_{0}$ : no mutation, 1: heterozygous, 2: homozygous for the C677T mutation. †Homocysteine. ‡intra-group comparison: baseline $v$ week 52. Sinter-group comparison.

with patients without any adverse event in the same time interval. No relation existed between the occurrence of adverse events overall and plasma homocysteine concentrations. Neither was there a relation between the baseline homocysteine and the occurrence of adverse events (Pearson's $r$ all $<0.3$ ). There were no relations between concentrations of serum folate at any time point or changes of these concentrations, baseline vitamin B12 concentrations, and the genotype of the MTHFR enzyme in itself on the one hand and occurrence of adverse events on the other.

\section{Discussion}

In this study different effects of SSZ, MTX, and the combination of the two were observed on plasma homocysteine concentrations. The two treatment groups receiving MTX showed a persistent increase in homocysteine, which was most pronounced in the COMBI group. No persistent influence of SSZ on plasma homocysteine existed, only in the early phase a significant but small effect occurred. Although a decrease in plasma folate explained some of the increase in plasma homocysteine, in a multivariate analysis an independent influence of MTX on plasma homocysteine has been demonstrated.

The lack of consistent effect of SSZ on plasma homocysteine in the first (longitudinal) study on this matter indicates that SSZ is not an efficient folate antagonist in vivo. This contradicts the results of an in vitro study ${ }^{3}$ that used suprapharmacological doses of SSZ, which might explain the discrepancy.

However, MTX induced a clear rise in plasma homocysteine, already discernable in the first weeks of treatment, and even clearly increasing up to six months of treatment. No significant changes were seen thereafter. This result differs from another study ${ }^{25}$ of plasma homocysteine in MTX treated RA patients with a follow up of 24 weeks, showing no influence of MTX on plasma homocysteine. In that study however, most evaluable patients received folic acid; the number of patients treated with MTX alone was only three. Also, blood was obtained in non-fasting state, which might have influenced the results. A recent article of the same research group described the influence of MTX and folic acid on plasma homocysteine in patients with MTX with or without folic acid supplementation. ${ }^{8}{ }^{26}$ In the MTX plus placebo group an increase in plasma homocysteine together with a decrease in plasma folic acid was observed in that study, in contrast with the folic acid treated patients in whom the reverse results were observed. The time curve of the increase in plasma homo- cysteine in our study during MTX treatment can best be explained by a gradual increase in concentration of intracellular polyglutamated methotrexate. ${ }^{27}$

We found an additional effect of SSZ when added to MTX. The difference with MTX alone did not reach statistical significance, possibly because of insufficient number of patients. It could be speculated that the weak and inconsistent influence of SSZ on folate metabolism becomes more important when this metabolism is hampered by MTX, resulting in an additive effect of SSZ. It is tempting to assume that this possible additional influence of SSZ is somehow related to the better clinical results found in one open study of a different group of patients with more advanced RA treated with the combination of SSZ and MTX, ${ }^{12}$ although the additional clinical efficacy of the combination was not demonstrated in the present study.

There was some influence of the MTHFR genotype on the impact of medication on the rise in plasma homocysteine: heterozygous patients had a higher plasma homocysteine after one year than the patients without a mutation. Patients homozygous for the mutation started with a high plasma homocysteine and remained high, without a significant change, possibly because of a ceiling effect. No significant differences existed between SSZ and MTX in this respect, although a lack of power because of a low number of patients in the various subgroups might obscure more subtle relations. Possibly, when the mutation is present in the heterozygous state and the enzyme is further impeded either directly by $\mathrm{SSZ}^{3}$ or indirectly by interference with folate metabolism by MTX, production of methyltetrahydrofolate will fall short with impeded methylation of homocysteine as a result.

No correlation between plasma homocysteine and clinical efficacy was found in this study. Two possible explanations exist: firstly, the number of patients studied was too low to detect subtle changes. Secondly, and more likely given the probable lack of influence of folic and folinic acid (see introduction), efficacy of MTX and SSZ is not mediated by methylation of homocysteine or other folate mediated methylation reactions, but by another pathway, for example, purine synthesis by inhibition of 5-aminoimidazole-4-carboxamide ribonucleotide transformylase, as was reviewed by Cronstein. ${ }^{28}$ Although no relation was found between the baseline plasma homocysteine and toxicity, which is in contrast with the study of Morgan $\mathrm{et} \mathrm{al},{ }^{21}$ there was a relation between the change of plasma homocysteine and the occurrence of side effects in this study, although this 
was limited to gastrointestinal toxicity. It is remarkable however that the patients treated with COMBI had the highest homocysteine levels and also had the highest occurrence of gastrointestinal side effects. The lack of relations with other adverse events might be because of the low number of patients in these categories. The absence of a relation of efficacy with plasma homocysteine and the presence of some relation with toxicity strengthens the notion of different pathways leading to efficacy and toxicity. In this respect we would like to postulate that the pathway involving homocysteine remethylation mediates toxicity. This could be further investigated by lowering homocysteine by betaine administration to patients experiencing toxicity, which will increase homocysteine remethylation by the folate independent betaine-homocysteine methyltransferase (see fig 1).

In this study no control group was incorporated, which might flaw the conclusions drawn. However, it is not ethical to withhold patients with an active RA from confirmed efficacious drugs. In a pilot study of five patients with more advanced RA treated with azathioprine we found a slight but not significant decrease in plasma homocysteine over a period of six months (change -0.6 ( SD 3.4) $\mu \mathrm{mol} / \mathrm{l}$ ).

Although not investigated in this study, it would be of interest to see whether serum methionine is lowered, reciprocal to the increase in plasma homocysteine. However, a pilot study of 21 patients treated with SSZ, MTX, and their combination and the above mentioned azathioprine patients revealed no change in methionine (0.0 (SD 5.8) $\mu \mathrm{mol} / \mathrm{l})$.

The impact of SSZ and MTX alone and in combination on the folate metabolism involving transmethylation processes needs to be studied further. The influence on efficacy and toxicity of SSZ and MTX mediated by the folate metabolism (by giving folates, and possibly betaine in pharmacological quantities), ultimately connecting it to inflammatory active substances (for example, polyamines, adenosine, and eventually molecules like cytokines) should be investigated more extensively. This could clarify those pathways leading to efficacy and toxicity and the possible difference in these pathways.

In conclusion, MTX but not SSZ leads to a consistent increase in plasma homocysteine concentrations. This increase was even more pronounced in the COMBI treated patients. The change in plasma homocysteine is related to the C677T mutation in the methylenetetrahydrofolate reductase gene. Plasma homocysteine did not relate with clinical efficacy, but a relation with gastrointestinal toxicity was observed.

We would like to thank Stephanie M G Vloet, Chris M G Thomas, and Frans J M Trijbels for their contribution to this study. Funding: this study was partly financed by grants of Pharmacia $\mathrm{AB}$, Sweden and "Het Nationaal Reumafonds", the Netherlands.

1 Felson DT, Anderson JJ, Meenan RF. Use of short-term efficacy/toxicity tradeoffs to select second-line drugs in rheumatoid arthitis. A metanalysis of published trials. Arthritis Rheum 1992;35:1117-25.
2 Bannwarth B, Labat L, Moride Y, Schaeverbeke T. Methotrexate in rheumatoid arthritis. An update. Drugs Methotrexate in
1994;47:25-50.

3 Selhub J, Dhar J, Rosenberg IH. Inhibition of folate enzymes by sulfasalazine. J Clin Invest 1978;61:221-4.

4 Bird HA. Sulphasalazine, sulphapyridine or 5 -aminosalicylic acid- Which is the active moiety in rheumatoid arthritis? Br J Rheumatol 1995;34 (suppl 2):16-19.

5 Franklin JL, Rosenberg IH. Impaired folic acid absorption in inflammatory bowel disease: effects of salicyazosulfapyridine (azulfidine). Gastroenterology 1973;64:517-25.

6 Grindulis KA, McConkey B. Does sulfasalazine cause folate deficiency in rheumatoid arthritis? Scand J Rheumatol 1985;14:265-70.

7 Tishler M, Caspi D, Fishel B, Yaron M. The effects of leucovorin (folinic acid) on methotrexate therapy in rheuma toid arthritis patients. Arthritis Rheum 1988;31:906-8.

8 Joyce DA, Will RK, Hoffman DM, Laing B, Blackbourn SJ. Exacerbation of rheumatoid arthritis in $p$ atients treated with methotrexate after administration of folinic acid. Ann Rheum Dis 1991;50:913-14.

9 Morgan SL, Babbott JE, Vaugh WH, Austin JS, Veitch TA, Lee JY, et al. Supplementation with folic acid during methotrexate therapy for rheumatoid arthritis. A double blind, placebo-controlled trial. Ann Intern Med 1994;121:83341 .

10 Hanrahan PS, Russell AS. Concurrent use of folinic acid and methotrexate in rheumatoid arthritis. J Rheumatol 1988;15:1078-80

11 Buckley LM, Vacek PM, Cooper SM. Administration of folinic acid after low dose methotrexate in patients with rheumatoid arthritis. J Rheumatol 1990;17:1158-61.

12 Weinblatt ME, Maier AL, Coblyn JS. Low dose leucovorin does not interfere with the efficacy of methotrexate in rheumatoid arthritis: an 8 week randomized placebo controlled trial. J Rheumatol 1993;20:950-2.

13 Shiroky JB, Neville C, Esdaile JM, et al. Low-dose methotrexate with leucovorin (folinic acid) in the management of rheumatoid arthritis. Results of a randomised, ment of rheumatoid arthritis. Results of a randomised, 1993;35:795-803.

14 Cronstein BN, Naime D, Ostad E. The antiinflammatory mechanism of methotrexate. Increased adenosine release at inflamed sites diminishes leukocyte accumulation in an in vivo model of inflammation. J Clin Invest 1993;92:2675-82.

5 Nesher G, Moore TL. The in vitro effects of methotrexate on peripheral blood mononuclear cells. Modulation by methyldonors and spermidine. Arthritis Rheum 1990;33:954-9.

16 Haagsma CJ, van Riel PLCM, van de Putte LBA. Combination of methotrexate and sulphasalazine versus methotrexate alone. A randomized open clinical trial in rheumatoid arthritis patients resistant to sulphasalazine therapy. Br J Rheumatol 1994;33:1049-55.

17 Refsum H, Helland S, Ueland PM. Fasting plasma homocysteine as a sensitive parameter of antifolate effect: a study of psoriasis patients receiving low-dose methotrexate reatment. Pharmacol Ther 1989;46:510-20.

18 Van der Put NMJ, Eskes TKAB, Blom HJ. Is the common $677 \mathrm{C}-\mathrm{T}$ mutation in the methylenetetrahydrofolate reductase a risk factor for neural tube defects? A mega-analysis. Q J Med 1997;90:111-15.

19 Frosst P, Blom HJ, Milos R, Goyette P, Sheppard CA, Matthews RG, et al. A candidate genetic risk factor for vascular disease: a common mutation in methylenetetrahydrofolate reductase. Nature Genetics 1995;10:111-13.

20 Van der Put NMJ, Steegers-Theunissen RPM, Frosst P, Trijbels FJM, et al. Mutated methylenetetrahydrofolate reductase as a risk factor for spina bifida. Lancet 1995;346: reductase $1070-1$.

21 Van der Heijde DMFM, van't Hoff MA, van Riel PLCM, Theunisse LAM, Lubberts EW, van Leeuwen MA, et al. Judging disease activity in clinical practice in rheumatoid arthritis: first step in the development of a disease activity score. Ann Rheum Dis 1990;49:916-20.

22 Te Poele-Pothoff MTWB, van den Berg M, Franken DG, Boers GHJ, Jakobs C, de Kroon IFI, et al. Three different methods for the determination of total homocysteine in plasma. Ann Clin Biochem 1995;32:218-20.

23 Mooij PNM, Thomas CMG, Doesburg WH, Eskes TKAB. Multivitamin supplementation in oral contraceptive users. Contraception 1991;44:277-88.

24 Haagsma CJ, van Riel PLCM, de Jong AJL, van de Putte LBA. Combination of sulphasalazine and methotrexate versus the single components in early rheumatoid arthritis: a randomized, controlled, double blind, 52 week clinical trial. Br J Rheumatol 1997;36:1082-8,

25 Morgan SL, Baggott JE, Refsum H, Ueland PE. Homocysteine levels in patients with rheumatoid arthritis treated with low-dose methotrexate. Clin Pharmacacol 1991;50: $547-56$

26 Morgan SL, Baggott JE, Lee JY, Alarcon GS. Folic acid supplementation prevents defecient blood folate levels and phentation prever hyperhomothcardiovascular disease prevention. J Rheumatol 1998;25: $441-6$

7 Chabner BA, Allegra CJ, Curt GA, Clendeninn NJ, Baram J, Koizumi S, et al. Poyglutamation of methotrexate: i methotrexate a prodrug? J Clin Invest 1985;76:907-12

28 Cronstein BN. Molecular therapeutics. Methotrexate and its mechanism of action. Arthritis Rheum 1996;39:1951-60. 